\title{
ANÁLISE DE SENSIBILIDADE DO SISTEMA DE DRENAGEM AGRÍCOLA-SDA MEDIANTE VARIAÇÃO DOS PARÂMETROS DE ENTRADA ${ }^{1}$
}

\author{
Sensibility analyses of the agricultural drainage systems-SDA through the variation \\ of the input parameters
}

\author{
Adão Wagner Pêgo Evangelista ${ }^{2}$,Paulo Afonso Ferreira ${ }^{3}$
}

\begin{abstract}
RESUMO
O modelo SDA foi desenvolvido com vistas ao dimensionamento e manejo de sistema de drenagem agrícola, em regiões de clima úmido e subúmido e fundamenta-se no critério de drenagem conhecido como somatório dos excessos de água no solo acima de $30 \mathrm{~cm}\left(\mathrm{SEW}_{30}\right)$, que tem-se mostrado eficaz para estas condições climáticas. Com este trabalho, contempla-se a realização da análise de sensibilidade do modelo SDA, objetivando verificar o efeito dos parâmetros de entrada sobre os resultados obtidos pela aplicação do modelo. As simulações realizadas com o modelo mostraram ser este bastante sensível aos parâmetros de entrada, apresentando alta sensibilidade à precipitação total diária, à profundidade de instalação dos drenos, à condutividade hidráulica do solo saturado e aos valores de evapotranspiração de referência.
\end{abstract}

Termos para indexação: Drenagem agrícola, modelo de simulação.

\begin{abstract}
The SDA model was developed in order to attend the design and agricultural drainage system management in humid and subhumid climate regions based on the drainage criteria known as the total amount of exceeding water on soil above 30 $\mathrm{cm}\left(\mathrm{SEW}_{30}\right)$ and has showed efficient for those climate conditions. This study aimed at accomplishing sensitivity analysis of SDA model, in order to verify the effect of the input parameters on the results obtained by application of the model. Simulations accomplished with the model showed to be very sensible to input parameters, showing high response to total daily precipitation, installation depth of drains, hydraulic conductivity of saturated soil and the reference evapotranspiration values.
\end{abstract}

Index terms: Agricultural drainage, simulation model.

(Recebido para publicação em 14 de janeiro de 2004 e aprovado em 10 de março de 2005)

\section{INTRODUÇÃO}

Ao se delinear um sistema integrado de manejo de água-solo-planta, deve-se considerar as propriedades físico-químicas do solo, a topografia, o clima, a cultura e os requerimentos de trafecabilidade. A metodologia a ser utilizada para projetar e analisar sistemas de manejo de água-solo-planta, deve permitir identificar seqüências de condições climáticas que podem ser críticas para a produção das culturas, além de descrever o desempenho do sistema durante certo período (SKAGGS, 1981).

O modelo SDA desenvolvido por Evangelista (2003) com base em séries sintéticas de dados diários de precipitação e evapotranspiração de referência, características físico-hídricas do solo, dados de sensibilidade da cultura ao excesso de água, profundidade do sistema radicular e características do sistema de drenagem, permite o dimensionamento e manejo de sistemas de drenagem agrícola, em regiões de clima úmido e subúmido. Segundo o autor, embora sejam necessárias informações de tolerância das culturas tanto ao excesso quanto ao déficit de água, o modelo enfatiza a tolerância ao excesso de água. A infiltração da água no solo é estimada a partir da equação de Green \& Ampt (1911) modificada por Mein \& Larson (1973). A posição do lençol freático é estimada pela equação de Krayjenhoff Van LeurMaasland. O efeito do espaçamento e profundidade dos drenos sobre a produtividade das culturas, são caracterizados com base nos valores do $\mathrm{SEW}_{30}$ calculados a partir do hidrograma freático, gerado durante o período de desenvolvimento da cultura.

\footnotetext{
1 Parte da tese apresentada à Universidade Federal de Viçosa/UFV, para obtenção do título de "Doctor Scientiae", pesquisa financiada pela FAPEMIG.

2 Bolsista recém-doutor, DEG - Universidade Federal de Lavras/UFLA - Cx. P. 3037 - 37.200-000 - Lavras, MG - awpego@ufla.br

${ }^{3}$ Professor Titular, DEA - Universidade Federal de Viçosa/UFV.
} 
Estudos de sensibilidade permitem verificar se um modelo produz resultados lógicos em função de alterações nos parâmetros de entrada. Resultados desses estudos fornecem informações sobre o comportamento do modelo e, também, sobre falhas na metodologia utilizada no desenvolvimento do modelo e na sua aplicabilidade a diferentes cenários e escalas (GOWDA et al., 1999).

Segundo Goldenfum et al. (1991), para modelos simples é possível obter expressões das saídas em função dos dados de entrada, expressando a sensibilidade do modelo como funções explícitas. No entanto, à medida que os modelos tornam-se mais complexos, a sensibilidade é expressa mais facilmente na forma de mudanças relativas, gráficos e tabelas, em vez de funções. O autor afirma que essa análise auxilia o usuário a determinar quais parâmetros devem ser estimados com precisão e quais não requerem grande precisão em suas estimativas.

Borges Júnior (2000) realizou a análise de sensibilidade do modelo PRODREN aos seus parâmetros de entrada. Os resultados mostraram que os parâmetros profundidade média dos drenos, condutividade hidráulica do solo saturado, porosidade drenável, parâmetros climáticos, coeficiente $\mathrm{CN}$ (Curve Number) e custo de instalação dos drenos subterrâneos exercem maior influência no cálculo do espaçamento econômico de drenos. Duarte (1997) realizou um estudo semelhante com o modelo SIMDRENO e os resultados da análise de sensibilidade revelaram que a profundidade dos drenos, a condutividade hidráulica do solo e os parâmetros hidrológicos da região foram os fatores cuja variação exerce maior efeito sobre o espaçamento mais econômico de drenos.

Face a necessidade de conhecimento dos parâmetros de entrada do modelo SDA que exercem maior influência no dimensionamento de sistema de drenagem agrícola, com o presente trabalho objetivou-se realizar a análise de sensibilidade do modelo SDA aos parâmetros de entrada.

\section{MATERIAL E MÉTODOS}

O aplicativo Delphi foi utilizado no desenvolvimento do modelo denominado SDA, desenvolvido por Evangelista (2003), visando o dimensionamento e manejo de sistemas de drenagem agrícola, no qual fundamenta-se no critério de drenagem $\mathrm{SEW}_{30}$ - somatório dos excessos de água no solo acima da profundidade de $30 \mathrm{~cm}$, no balanço de água no solo e na equação de drenagem de Krayjenhoff van Leur-Maasland.
Para obtenção do índice $\mathrm{SEW}_{30}$, são realizados um balanço hídrico diário no solo, na região do sistema radicular e o cálculo da posição diária do lençol freático.

Os componentes do balanço hídrico (Figura 1) são contabilizados diariamente em um volume de controle de base unitária, localizado eqüidistante a dois drenos paralelos, estendendo-se desde a camada impermeável até a superfície do solo. Estes componentes são: o total precipitado diário, a infiltração, o armazenamento de água na zona radicular, o fluxo ascendente máximo proveniente do lençol freático para a zona radicular, a evapotranspiração diária e a drenagem.

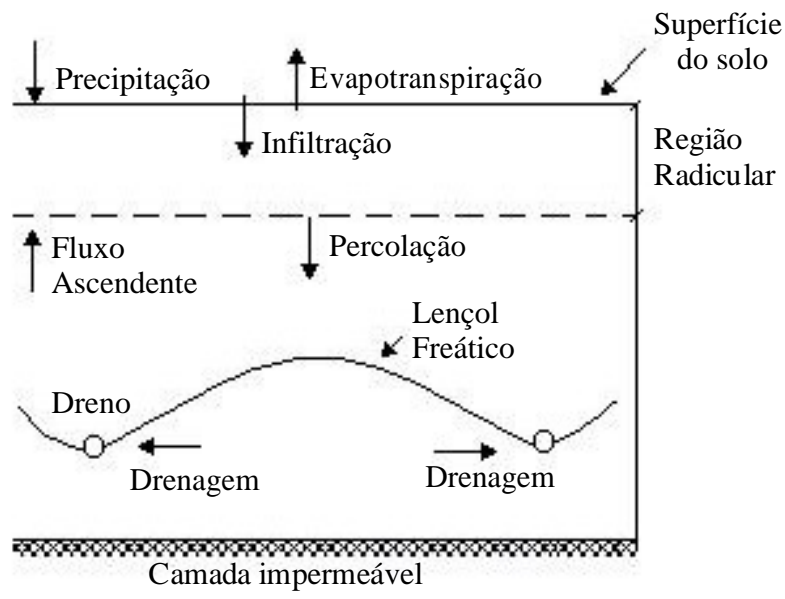

FIGURA 1 - Componentes do balanço hídrico considerados no modelo DAS.

O balanço de água na zona radicular é realizado, considerando-se a fração da precipitação pluvial diária, ou lâmina de irrigação, infiltrada, que efetivamente provoca a recarga do lençol freático, a evapotranspiração real diária e o fluxo ascendente máximo proveniente do lençol freático.

A posição diária do lençol freático (Figura 2) em resposta às recargas efetivas é calculada utilizando-se a equação de recarga intermitente de Krayjenhoff van LeurMaasland, durante o ciclo da cultura, para uma determinada profundidade dos drenos e coeficiente de armazenamento.

Os efeitos da relação entre a profundidade do lençol freático e a produtividade da cultura são caracterizados com base no valor de $\mathrm{SEW}_{30}$, calculado a partir do hidrograma freático, gerado durante o período de desenvolvimento da cultura (Figura 2). Numa segunda etapa, o valor calculado de $\mathrm{SEW}_{30}$ é comparado com valores

Ciênc. agrotec., Lavras, v. 30, n. 1, p. 133-139, jan./fev., 2006 
experimentais determinados para a cultura e tipo de solo em questão. Quando o valor calculado de $\mathrm{SEW}_{30}$ não coincide com os experimentais, um novo valor de espaçamento é testado, de forma iterativa, até obter-se uma diferença aceitável.

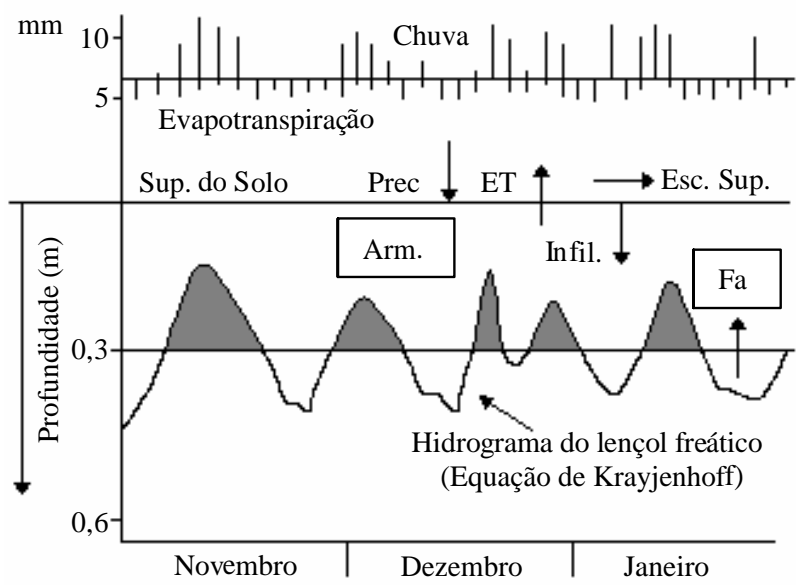

FIGURA 2-Hidrograma freático e índice $\mathrm{SEW}_{30}$.

Os parâmetros de entrada do modelo são: precipitação total diária, mm; duração da precipitação, h; intensidade máxima de precipitação padronizada, adimensional; tempo padronizado de ocorrência da intensidade máxima de precipitação, adimensional; temperatura máxima do ar, ${ }^{0} \mathrm{C}$; temperatura mínima do ar, ${ }^{0} \mathrm{C}$; temperatura do ponto de orvalho, ${ }^{0} \mathrm{C}$; radiação solar, $\mathrm{MJ} \mathrm{m}^{-2} \mathrm{~d}^{-1}$; velocidade do vento a $2 \mathrm{~m}$ de altura, $\mathrm{m} \mathrm{s}^{-1}$; condutividade hidráulica do solo saturado, $\mathrm{m} \mathrm{d}^{-1}$; profundidade da camada impermeável, m; valor inicial de espaçamento entre drenos, $\mathrm{m}$; profundidade de instalação dos drenos, $\mathrm{m}$; raio efetivo dos drenos, m; curva de retenção de água no solo; data do plantio e colheita da cultura; valor de $\mathrm{SEW}_{30}$ experimental, $\mathrm{cm} . d$; profundidade efetiva do sistema radicular da cultura, $\mathrm{m}$ e teor de água no solo no dia inicial, adimensional. As etapas descritas acima são esquematizadas no fluxograma apresentado no Anexo 1.

Após a simulação, o programa apresenta os seguintes parâmetros de saída: $\mathrm{SEW}_{30}$ calculado, cm.d; produtividade relativa em função do excesso de água no solo, decimal e espaçamento entre drenos paralelos, $\mathrm{m}$.

\section{Análise de sensibilidade}

A análise de sensibilidade do modelo foi realizada variando-se cada parâmetro de entrada, individualmente, enquanto os outros eram mantidos constantes, sendo conduzida para os parâmetros relativos à geometria do sistema de drenagem, às características físico-hídricas do solo, aos elementos meteorológicos e à sensibilidade da cultura ao déficit de água no solo. Para quantificar o impacto de um intervalo de valores de um dado parâmetro de entrada sobre os resultados obtidos com o modelo, foi utilizado o índice de sensibilidade relativo (IS), determinado conforme McCuen \& Snyder (1986), pela equação:

$$
I S=\frac{\frac{R_{1}-R_{2}}{R_{12}}}{\frac{I_{1}-I_{2}}{I_{12}}}
$$

em que:

IS = índice de sensibilidade do modelo aos parâmetros de entrada;

$\mathrm{R}_{1}=$ resultado obtido com o modelo para o menor valor de entrada;

$\mathrm{R}_{2}=$ resultado obtido com o modelo para o maior valor de entrada;

$\mathrm{R}_{12}=$ média dos resultados obtidos com o menor e o maior valores de entrada;

$I_{1}=$ menor valor de entrada;

$\mathrm{I}_{2}=$ maior valor de entrada; $\mathrm{e}$

$\mathrm{I}_{12}=$ média dos valores de entrada.

Conforme McCuen \& Snyder (1986), o valor de IS representa a mudança normalizada gerada na saída do modelo para uma mudança normalizada na entrada dos dados, a qual permite comparar a sensibilidade a diferentes magnitudes dos parâmetros de entrada, representando uma função dos parâmetros de entrada para uma resposta nãolinear. Quanto maiores (em módulo) forem os índices obtidos, mais sensível será o modelo ao parâmetro, já os valores próximos a zero indicam que o modelo não apresenta sensibilidade ao parâmetro.

\section{Base de dados}

Para realização da análise de sensibilidade, utilizouse a cultura da cana-de-açúcar e séries sintéticas de 50 anos de elementos meteorológicos gerados pelo programa 
CLIGEN (NICKS et al., 1995) para a região de Viçosa, MG. A metodologia utilizada no modelo CLIGEN para gerar os elementos meteorológicos é descrita em Nicks et al. (1995). O tipo de solo foi o Latossolo Vermelho-Amarelo e o valor de $\mathrm{SEW}_{30}$ para a cana-de-açúcar utilizado foi o obtido por Skaggs (1981), ou seja, $242 \mathrm{~cm}$.d. A evapotranspiração de referência $\left(\mathrm{ET}_{0}\right)$ foi estimada aplicando-se o modelo proposto por Penman - Monteith padrão FAO, conforme Allen et al. (1998), utilizando os elementos meteorológicos gerados pelo CLIGEN.

Na Tabela 1, apresenta-se os valores dos parâmetros geométricos do sistema de drenagem e os físicohídricos do solo utilizados na análise de sensibilidade. $\mathrm{Na}$ Tabela $1, \mathrm{~m}$ h e b são parâmetros do modelo de Van
Genuchten (1980) e para a determinação da porosidade drenável utilizou-se a umidade em equilíbrio com o potencial matricial de $6 \mathrm{kPa}$.

\section{RESULTADOS E DISCUSSÃO}

Os índices de sensibilidade obtidos mostram que o modelo é bastante sensível a alguns dos parâmetros estudados, indicando que pequenas alterações nesses parâmetros resultam em diferenças expressivas nos resultados apresentados pelo modelo. Portanto, esses parâmetros necessitam de uma maior precisão ao serem determinados. Na Tabela 2 é apresentada uma síntese dos índices de sensibilidade obtidos para os diferentes parâmetros analisados.

TABELA 1 - Parâmetros físico-hídricos do solo e geométricos do sistema de drenagem, utilizados na análise de sensibilidade.

\begin{tabular}{lc}
\hline Parâmetros & Valor \\
\hline Condutividade hidráulica do solo saturado & $1,00 \mathrm{~m} \mathrm{~d}^{-1}$ \\
Porosidade drenável & $0,07 \mathrm{~m}^{3} \mathrm{~m}^{-3}$ \\
Umidade volumétrica do solo saturado & $0,31 \mathrm{~m}^{3} \mathrm{~m}^{-3}$ \\
Umidade volumétrica do solo na capacidade de campo & $0,24 \mathrm{~m}^{3} \mathrm{~m}^{-3}$ \\
Umidade vol. do solo no ponto de murcha permanente & $0,21 \mathrm{~m}^{3} \mathrm{~m}^{-3}$ \\
Umidade volumétrica do solo no dia inicial & $0,25 \mathrm{~m}^{3} \mathrm{~m}^{-3}$ \\
Umidade volumétrica residual & $0,20 \mathrm{~m}^{3} \mathrm{~m}^{-3}$ \\
$\mathrm{~m}$ & 0,32 \\
$\eta$ & 1,47 \\
$\beta$ & $0,10 \mathrm{~cm}^{-1}$ \\
Profundidade do sistema radicular & $1,10 \mathrm{~m}$ \\
Profundidade do perfil (camada impermeável) & $5 \mathrm{~m}$ \\
Profundidade dos drenos & $1,4 \mathrm{~m}$ \\
Raio efetivo dos drenos & $0,05 \mathrm{~m}$ \\
\hline
\end{tabular}

$\mathrm{m}$ e h são parâmetros adimensionais.

TABELA 2 - Valores dos índices de sensibilidade obtidos para diferentes parâmetros de entrada.

\begin{tabular}{|c|c|c|}
\hline Parâmetro de entrada & $\begin{array}{l}\text { Intervalo de } \\
\text { valores }\end{array}$ & $\begin{array}{c}\text { Índice de } \\
\text { Sensibilidade }\end{array}$ \\
\hline Precipitação (mm) & -40 a $160 \%{ }^{*}$ & $-1,014$ \\
\hline Profundidade dos drenos (m) & $0,84-2,52$ & 0,85 \\
\hline Condutividade hidráulica $\left(\mathrm{m} \mathrm{d}^{-1}\right)$ & $0,10-1,60$ & 0,81 \\
\hline Profundidade da camada de impedimento (m) & $2-10$ & 0,68 \\
\hline Evapotranspiração de referência $\left(\mathrm{mm} \mathrm{d}^{-1}\right)$ & -90 a $60 \% *$ & 0,27 \\
\hline $\mathrm{SEW}_{30}(\mathrm{~cm} . \mathrm{d})$ & $96,80-387,2$ & $-0,26$ \\
\hline Porosidade drenável & $0,023-0,1252$ & 0,22 \\
\hline Raio efetivo dos drenos (mm) & $30-130$ & 0,09 \\
\hline
\end{tabular}

Faixa de variação dos elementos.

Ciênc. agrotec., Lavras, v. 30, n. 1, p. 133-139, jan./fev., 2006 
De acordo com os valores apresentados no Tabela 2, o modelo apresenta maior sensibilidade à precipitação, seguido da profundidade de instalação dos drenos, da condutividade hidráulica do solo saturado, da profundidade da camada de impedimento e da evapotranspiração de referência. $\mathrm{O}$ menor valor do índice de sensibilidade foi obtido para o raio efetivo dos drenos.

Os efeitos da variação dos parâmetros de entrada sobre os resultados das simulações obtidos com a aplicação do modelo são apresentados nas Figuras 3 e 4 .

Na Figura 3, observa-se que subestimavas da precipitação e superestimavas da evapotranspiração de referência (ET) promoveram um aumento sensível do espaçamento entre drenos. Esses resultados estimulam o uso de modelos mais precisos para a estimativa da ET e para a geração de séries sintéticas de precipitação. Percebe-se também que o modelo é altamente sensível a variações negativas no valor da precipitação total diária, o que pode ser evidenciado pela alta declividade da função que representam as variações da precipitação neste trecho considerado. Este fato já era esperado, pois, quanto menor for a lâmina precipitada, a tendência natural é a de utilização de espaçamentos menores. No entanto, para variações positivas nos valores de precipitação, nota-se que a declividade da função é bem menor quando comparada ao caso anterior, ou seja, pequenas variações nos valores de precipitação refletem em menores variações nos valores do espaçamento. Este fato pode ser explicado pela consideração do efeito do escoamento superficial sobre o espaçamento.

Variações positivas no fator de sensibilidade da cultura ao excesso de água no solo acarretam subestimativa do espaçamento entre drenos, o que evidencia a necessidade de pesquisas com o objetivo de determinação adequada deste fator, para diferentes variedades de culturas.

Na Figura 4, observam-se os efeitos da variação dos parâmetros de entrada relativos à geometria do sistema de drenagem e às características físico-hídricas do solo sobre os resultados das simulações obtidos com a aplicação do modelo. Verificou-se que as variações positivas desses parâmetros promoveram o aumento do espaçamento entre drenos. A profundidade de instalação dos drenos foi o parâmetro que propiciou o maior efeito, incentivando a realização de estudos visando a determinação da profundidade adequada de instalação dos drenos. O modelo também foi bastante sensível a variações da condutividade hidráulica e, em escala bem menor, às variações da porosidade drenável. Esses resultados estão

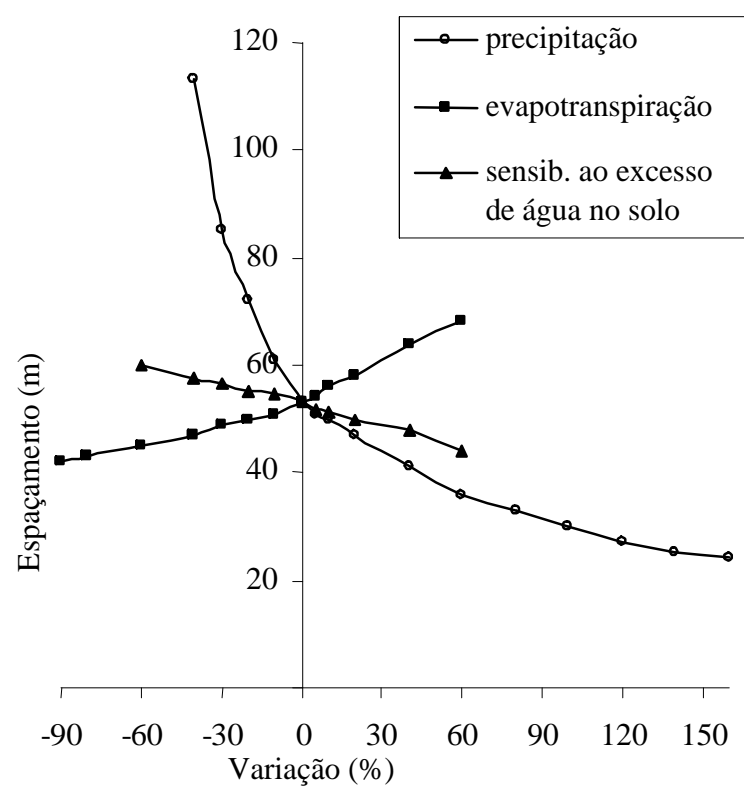

FIGURA 3 - Sensibilidade do modelo aos parâmetros hidrológicos e da cultura.

em concordância com as observações de Ferreira (2003), segundo os quais a condutividade hidráulica deve ser determinada de forma mais criteriosa quando comparada com a determinação da porosidade drenável. Percebe-se também que determinações incorretas da profundidade do perfil do solo passam a ter efeito pequeno à medida em que esse parâmetro aumenta. Esse resultado torna menos importante a realização de sondagens mecânicas profundas visando a localização precisa da camada de impedimento.

Analisando-se ainda a Figura 4, percebe-se que as funções que representam as variações dos parâmetros profundidade de instalação dos drenos e condutividade hidráulica do solo saturado apresentam uma mesma tendência e declividades. Evidencia-se que pequenas variações nestes valores causam pequenas variações nos valores dos espaçamentos. Da mesma forma, quando se comparam os efeitos das variações positivas dos parâmetros, profundidade da camada impermeável e porosidade drenável, sobre o espaçamento dos drenos, nota-se também que variações da mesma magnitude destes parâmetros exercem efeitos semelhantes sobre o espaçamento. Destaca-se também que estas funções tendem para uma assíntota horizontal, ou seja, a função atinge um ponto de máxima, sendo que, neste trecho, as variações nos parâmetros passam a não influenciar as alterações no valor do espaçamento. 


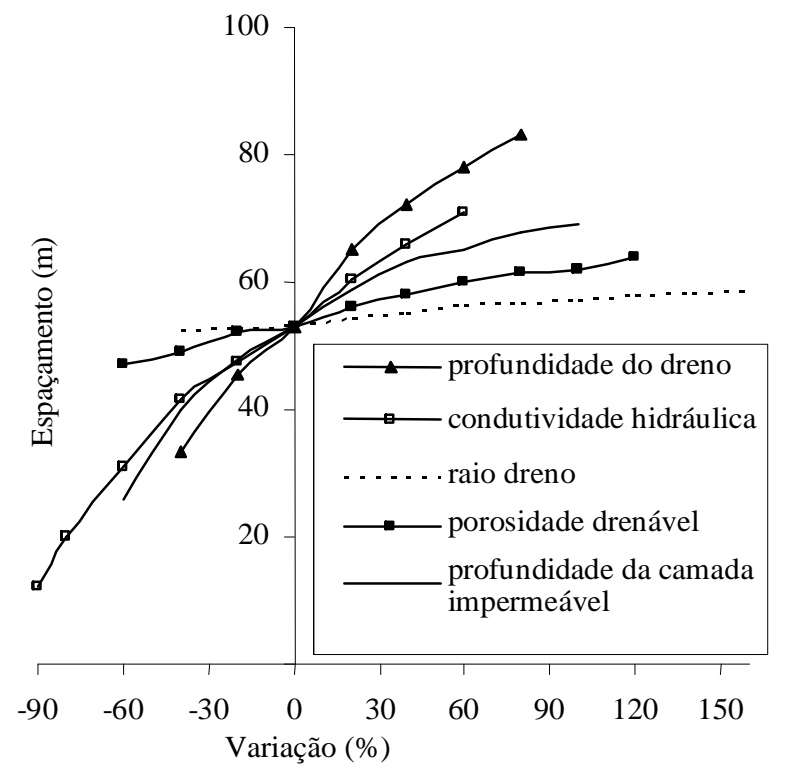

FIGURA 4 - Sensibilidade do modelo aos parâmetros relativos à geometria do sistema de drenagem e às características físico-hídricas do solo.

Para variações negativas dos parâmetros de entrada relativos à geometria do sistema de drenagem e às características físico-hídricas do solo, observa-se que o modelo mostrou-se mais sensível, em ordem, às variações nos parâmetros profundidade dos drenos, profundidade do perfil e condutividade hidráulica do solo saturado.

De maneira geral, verifica-se, pelos resultados da análise de sensibilidade, que o modelo produziu resultados lógicos em função de alterações nos seus parâmetros de entrada, comprovando, dessa forma, que a metodologia desenvolvida, em última análise, não contém falhas e pode ser aplicada a diferentes cenários e escalas.

\section{CONCLUSÕES}

a) O modelo SDA produziu resultados lógicos em função de alterações nos parâmetros de entrada;

b) Para a utilização de modelos é importante determinar com precisão os parâmetros de entrada a fím de obter resultados satisfatórios;

c) A análise de sensibilidade revelou que a profundidade dos drenos, a precipitação total diária, a condutividade hidráulica do solo saturado e a profundidade da camada impermeável são os parâmetros de entrada do modelo SDA que devem ser determinados com maior precisão.

\section{REFERÊNCIAS BIBLIOGRÁFICAS}

ALLEN, R.; PEREIRA, L. S.; RAES, D.; SMITH, M. Guidelines for predicting crop water requirements. Rome: FAO, 1998. 319 p. (Irrigation and Drainage Paper, 56).

BORGES JÚNIOR, J. C. F. Modelo computacional para dimensionamento e manejo de sistemas de drenagem agrícola. 2000. 106 f. Dissertação (Mestrado em Engenharia Agrícola) - Universidade Federal de Viçosa, Viçosa, 2000.

DUARTE, S. N. Modelo para avaliação de desempenho de sistemas de drenagem subterrânea e cálculo de espaçamento de drenos. 1997. 143 f. Tese (Doutorado em Engenharia Agrícola) - Universidade Federal de Viçosa, Viçosa, 1997.

EVAngelistA, A. W. P. Metodologia para dimensionamento e manejo de sistemas de drenagem agrícola. 2003. 106 f. Tese (Doutorado em Engenharia Agrícola) - Universidade Federal de Viçosa, Viçosa, 2003.

FERREIRA, P. A. Drenagem de terras agrícolas. Brasília, DF: ABEAS, 2003. 210 p.

GENUCHTEN, M. T. van. A closed equation for predicting the hydraulic conductivity of unsaturated soils. Soil Science Society American Journal, Madison, v. 44, n. 3, p. 892-898, 1980.

GOLDENFUM, J. A.; SEMMELMANN, F. R.; REICHERT, G. Produção de sedimentos em bacias rurais: análise de sensibilidade dos modelos answers, creams e mulsed. In: SIMPÓSIO LUSO-BRASILEIRO DE HIDRÁULICA E RECURSOS HÍDRICOS, 5.; SIMPÓSIO BRASILEIRO DE RECURSOS HÍDRICOS, 9., 1991, Rio de Janeiro. Anais... Rio de Janeiro: [s.n.], 1991.

GOWDA, P.; WARD, A.; WHITE, J. L.; DESMOND, E. The sensitivity of ADAPT model predictions of stream flows to parameters used to define hydrologic response units. Transactions of the ASAE, Saint Joseph, v. 42, n. 2, p. 381-389, 1999.

GREEN, W. H.; AMPT, G. A. Studies on soil physics-1: the flow of air and water through soils. Journal of Agricultural Science, Cambridge, v. 4, n. 1, p. 1-24, 1911. 
MCCUEN, R. H.; SNYDER, W. M. Hydrologic modeling: statistical methods and applications. Englewood Cliffs: Prentice Hall, 1986.

MEIN, R. G.; LARSON, C. L. Modeling infiltration during a steady rain. Water Resources Research, [S.1.], v. 9, n. 4, p. 384-394, 1973.
NICKS, A. D.; LANE, L. J.; GANDER, G. A. Weather generator: USDA-water erosion prediction project (WEPP). West Lafayette: USDA-ARS, 1995. cap. 2, 22 p.

SKAGGS, R. W.DRAINMOD - reference report: methods for design and evaluation of drainage-water management systems for soils high water tables. Raleigh: USDA-SCS, 1981. 329 p. 